drug producers, given that over 10000 bioactive metabolites have been recovered from these filamentous bacteria. Streptomyces species living in the dynamic mangrove forest are placed under constant pressure to thrive in such harsh environment, which is suggested to promote the production of interesting bioactive metabolites with anticancer properties. This project aims to investigate the cytotoxic and antioxidant activities of an extract derived from novel Streptomyces species isolated from mangrove forest in Malaysia.

Methods Four novel Streptomyces species (designated as MUSC $26^{\mathrm{T}}$, MUSC $136^{\mathrm{T}}$, MUSC $149^{\mathrm{T}}$ and MUSC $164^{\mathrm{T}}$ ) were identified from the poorly explored mangrove sediment (East Coast, Peninsular Malaysia) using polyphasic approach. As an attempt to explore the bioactive potential of these mangrovederived streptomycetes, extracts of these strains were prepared via fermentation and chemical extractions before performing in vitro biochemical and screening assays using cancer cell lines.

Results All of the methanolic extracts of these strains were shown to possess significant antioxidant activities. Among these strains, strain MUSC $136^{\mathrm{T}}$ displayed highest cytotoxic activity against colon cancer cell line HCT-116, killing more than half of them at $400 \mathrm{ug} / \mathrm{mL}$. In order to understand the mechanisms of actions involved, the levels of intracellular glutathione $(\mathrm{GSH})$ was evaluated as this ubiquitous non-protein thiol is crucial for cell survival. A drastic increase in the proportion of cells undergoing GSH depletion was observed higher $(44.11 \% \pm 6.21 \%)$ as compared to control. Along with this observation, higher expression level of tumour suppressor protein, p53 was observed in cells treated with MUSC $136^{\mathrm{T}}$ extract. Thus, we postulate that treatment of MUSC $136^{\mathrm{T}}$ extract might potentially trigger the activation of p53-dependent apoptosis pathways (figure 1).

Conclusions Altogether, these findings highlight the importance of novel strain discovery from the underexplored areas, like mangrove forest, particularly in search of chemopreventive agents.

\section{IDDF2018-ABS-0208 FAECAL HSF2 CONCENTRATION MAYBE USED AS AN EVALUATION INDEX FOR PREDICTING THE MUCOSAL HEALING OF UC}

Junkun Niu*. Department of Gastroenterology, The First Affiliated Hospital of Kunming Medical University, Kunming, China

\subsection{6/gutjil-2018-IDDFabstracts.29}

Background In our previous study, we found that Heat Shock transcription Factor 2 (HSF2) was differentially expressed in Ulcerative Colitis (UC) patients and increased in parallel with the severity of UC. So this study is aiming at providing a new noninvasive index for predicting the mucosal healing of UC.

Methods Faecal samples were collected from 51 UC with MES $(0,1,2,3)$ and health controls. The concentration of feacl HSF2 was detected by Elisa. The correlation between faecal HSF2 levels and MES was compared by Pearson correlation analysis. A total of 231 follow-up UC patients were included. The faecal samples were collected in the early morning, and colonoscopy was performed in the next day for MES scoring. MES.

Results The concentration of faecal HSF2 in the normal control group and UC patients with $\mathrm{MES}=0,1,2,3$ were $(0.64$ $\pm 0.09, \quad 1.30 \pm 0.35, \quad 1.84 \pm 0.46,2.38 \pm 0.57,3.38 \pm 0.42) \quad \mathrm{ng} / \mathrm{ml}$ respectively. The level of faecal HSF2 was a positive correlation with MES $(r=0.81)$. The concentration of faecal HSF2 was increasing in the group of UC patients with MES $=0,1,2,3$ compared with the normal control group. The sensitivity, specificity, positive and negative predictive value of faecal HSF2 to predict mucosal healing was $(67.8 \%, 80.9 \%, 67.1 \%$ and $81.5 \%$ ) respectively. The clinical value of specificity and the negative predictive value was better than sensitivity and positive predictive value. The AUC of faecal HSF2 to predict mucosal healing was 0.919 (95\% CI:0.846-0.992, p<0.0001). The AUC was greater than 0.9 that indicated the faecal HSF2 had a high accuracy to predict mucosal healing of UC.

Conclusions Faecal HSF2 concentration may be used as a high accuracy noninvasive evaluation index for predicting the mucosal healing of UC.

\section{IDDF2018-ABS-0226 CHARACTERISATION OF GENOMIC ALTERATIONS IN PROXIMAL AND DISTAL COLORECTAL CANCER PATIENTS}

${ }^{1}$ Ryia Illani Mohd Yunos*, ${ }^{1}$ Nurul Syakima Ab Mutalib, ${ }^{2}$ Janice Khor Sheau Sean, ${ }^{1}$ Sazuita Saidin, ${ }^{1}$ Mohd Ridhwan Abd Razak, ${ }^{1}$ Norshahidah Mahamad Nadzir, ${ }^{1}$ Zuraini Abd Razak, ${ }^{3}$ Isa Md Rose, ${ }^{4}$ Ismail Sagap, ${ }^{4}$ Luqman Mazlan. ${ }^{1}$ UKM Medical Molecular Biology Institute (UMBI), Universiti Kebangsaan Malaysia, Kuala Lumpur, Malaysia; ${ }^{2}$ Life Sciences Solutions, Thermo Fisher Scientific Inc., Singapore; ${ }^{3}$ Department of Pathology, Universiti Kebangsaan Malaysia, Kuala Lumpur, Malaysia; ${ }^{4}$ Department of Surgery, Universiti Kebangsaan Malaysia, Kuala Lumpur, Malaysia

\subsection{6/gutjnl-2018-IDDFabstracts.30}

Background Majority of the proximal colorectal cancer (CRC) patients are presented with advanced disease at diagnosis. Little is known about the differences in the genomic landscape between proximal and the more common distal CRCs. The objectives of this study are to investigate the somatic single nucleotide variants (SNV) and the hypothetically affected cellular pathways between the proximal and distal CRC patients. Methods Whole exome sequencing was performed on DNA extracted from 10 paired cancer-normal adjacent fresh frozen tissues using the Ion Proton platform. Sanger sequencing was performed to validate the variants identified in proximal and distal CRCs. In addition, in silico validation was performed on 619 CRC patients from The Cancer Genome Atlas (TCGA) study.

Results We obtained a total of 4835 and 4177 variants in proximal and distal CRC, respectively. In proximal CRC, 539 were protein-altering variants in 508 genes while the distal CRCs had 245 protein-altering mutations in 180 genes. The proximal CRCs showed significantly more protein-altering variants as compared to distal CRCs ( $\mathrm{p}$ value $=0.0001$ ). We performed a comparison between mutation frequency in proximal versus distal CRCs from 619 TCGA patients and validated 37 predominantly altered genes in proximal CRCs. We observed that $90 \%(n=9)$ of the CRC patients shared an affected Wnt signalling pathway with five genes being altered (12 